\title{
Transvesical Endoscopic Peritoneoscopy: A Novel 5 mm Port for Intra-Abdominal Scarless Surgery
}

\author{
Estevao-Lima, Carla Rolanda, José M. Pêgo, Tiago Henriques-Coelho, David Silva, \\ José L. Carvalho and Jorge Correia-Pinto* \\ From the Life and Health Sciences Research Institute, School of Health Sciences, University of Minho (EL, CR, JMP, DS, JCP), \\ and Departments of Gastroenterology (CR) and Anesthesiology (JMP), Sao Marcos Hospital, Braga and Department of Urology, \\ Santo António General Hospital (EL) and Department of Pediatric Surgery, Sao Joao Hospital (THC, JLC, JCP), Porto, Portugal
}

Purpose: Recently various groups reported successful attempts to perform intra-abdominal surgery through a transgastric pathway. We assessed the feasibility and safety of a novel transvesical endoscopic approach to the peritoneal cavity through a $5 \mathrm{~mm}$ port in a porcine model.

Materials and Methods: Transvesical endoscopic peritoneoscopy was performed in 8 anesthetized female pigs, including 3 nonsurvival and 5 survival animals. Under cystoscopic guidance a vesical hole was created on the ventral bladder wall with an open-ended ureteral catheter. An over tube with a luminal diameter of $5.5 \mathrm{~mm}$ was placed in the peritoneal cavity, guided by a 0.035-inch guidewire. In all animals we performed peritoneoscopy of the entire abdomen as well as liver biopsy and falciform ligament section. A vesical catheter was placed for 4 days in all survival animals, which were sacrificed by day 15 postoperatively.

Results: After a learning curve in the first 3 nonsurvival animals the creation of a vesical hole and placement of the over tube were performed without complication in all survival animals. In these animals we easily introduced an EndoEYE ${ }^{\mathrm{TM}}$ into the peritoneal cavity, which provided a view of all intra-abdominal viscera, as well as a $9.8 \mathrm{Fr}$ ureteroscope, which allowed simple surgical procedures without complications. In survival experiments all pigs recovered. Necropsy examination revealed complete healing of the vesical hole and no signs of infection or adhesions into the peritoneal cavity.

Conclusions: Transvesical endoscopic peritoneoscopy was technically feasible and it could be safely performed in a porcine model. This study provides encouragement for additional preclinical studies of transvesical surgery with or without combinations with other natural orifices approaches to design new intra-abdominal scarless procedures in what seems to be third generation surgery.

Key Words: swine, endoscopy, laparoscopy, peritoneal cavity

$\mathrm{S}$ ince the late 1980s, a revolution has begun with the implementation of laparoscopic techniques that have gained progressive acceptance as the gold standard for an increasing number of intra-abdominal procedures. ${ }^{1}$ In fact, there are many advantages to the laparoscopic approach, such as smaller incisions, decreased postoperative pain and more rapid patient recovery.

Recently Kalloo et al described a new port to the peritoneal cavity through a transgastric approach in a porcine model. ${ }^{2}$ Subsequently various groups described more complex intra-abdominal procedures, such as fallopian tube ligation, cholecystectomy and cholecystogastric anastomosis, gastrojejunostomy, partial hysterectomy and oophorectomy. ${ }^{3-6}$ The continuous evolution of new instruments and the unexpected success reported by these investigators in porcine

\footnotetext{
Submitted for publication September 17, 2005.

Study received approval from the ethical review boards, Minho University, Braga, Portugal.

Supported by Grants Bolsa de Investigação Básica JABA 2005 da Associação Portuguesa de Urologia and POCTI/SAU-OBS/56428/ 2004 from FCT-Portugal.

* Correspondence: Instituto de Ciências da Vida e da Saúde, Escola de Ciências da Saúde, Universidade do Minho, Campus de Gualtar, 4709-057 Braga, Portugal (telephone: +351 253604 807; FAX: +351 253604 831; e-mail: jcp@ecsaude.uminho.pt).
}

models with transgastric surgery seems to have opened a new era in the surgical field, that is endoscopic transvisceral surgery. ${ }^{7-11}$

In this sequence the development of new transvisceral approaches remains to be defined. In this pilot study we assessed the feasibility and safety of the transvesical endoscopic approach to the peritoneal cavity with liver biopsy and falciform ligament section in a porcine model.

\section{MATERIALS AND METHODS}

This study was approved by the ethical review boards of Minho University, Braga, Portugal. Transvesical procedures were performed in 8 female pigs, including 3 in nonsurvival studies. Survival studies were done in 5 consecutive 35 to 45 $\mathrm{kg}$ female pigs (Sus scrofus domesticus). These 5 pigs were followed after surgery for 15 days before sacrifice and necropsy examination.

\section{Pig Preparation}

The animals were withdrawn from food for 24 hours and from water for 6 hours before the surgical procedure. All procedures were performed using general anesthesia with $6.0 \mathrm{~mm}$ endotracheal intubation with a Ruschelit@ Super 
Safety Clear Tracheal Tube and mechanical ventilation. Pre-anesthesia medication consisted of intramuscular injection of $32 \mathrm{mg} / \mathrm{ml}$ azaperone (Stressnil@) reconstituted with 1 $\mathrm{mg} / \mathrm{ml}$ midazolam (Dormicum®) at dose of 0.15 to $0.2 \mathrm{ml} / \mathrm{kg}$.

Venous access was obtained through an intravenous line placed in the marginal ear vein. Anesthesia was induced with $3 \mu \mathrm{g} / \mathrm{kg}$ fentanyl (Fentanest®), $10 \mathrm{mg} / \mathrm{kg}$ thiopental sodium (Pentothal $@$ ) and $0.2 \mathrm{mg} / \mathrm{kg}$ vecuronium (Norcuron®). For infection prophylaxis all animals received an intramuscular injection of $1 \mathrm{gm}$ ceftriaxone (Rocephin®) before endoscopy. Anesthesia was maintained with $1.5 \%$ to $2 \%$ sevoflurane (Sevorane®) and a perfusion of $1 \mathrm{mg} / \mathrm{kg}$ per hour vecuronium (Norcuron®).

\section{Surgical Technique}

An Olympus® A2281 cystoscope with an A22001A telescope was introduced through the urogenital sinus and urethra into the bladder with hydrodistention. Before any further procedure the bladder was emptied of urine and refilled with saline. The vesicotomy site was selected on the ventral bladder wall caudal to the bladder dome. Figure 1 shows the procedures. The cystoscope was then replaced by an Olympus® A2942A ureteroscope guided through a Terumo® guidewire to achieve the bladder. A mucosal incision was made with Olympus ${ }^{\circledR}$ A2576 scissors introduced by the working channel of the ureteroscope. Subsequently a $5 \mathrm{Fr}$ Selectip ${ }^{\mathrm{TM}}$ open-ended ureteral catheter was pushed forward through the incision into the peritoneal cavity (fig. 2).

A 0.035-inch flexible tip Terumo® guidewire was then inserted into the peritoneal cavity through the ureteral catheter lumen. Guided by the flexible tip guidewire the vesical hole was enlarged with the dilator of a ureterorenoscope sheath (Microvasive-Boston Scientific Corp., Natick, Massa-
A
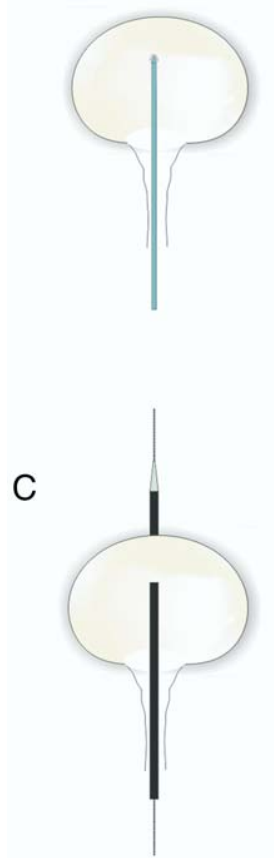

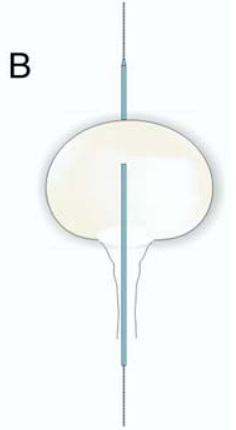

$\mathrm{D}$

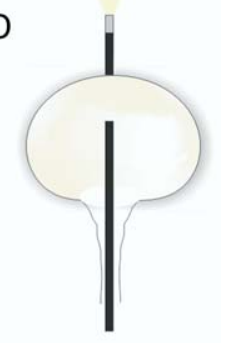

FIG. 1. $A$, vesical perforation with open-ended ureteral catheter, $B$, guidewire passage. $C$, placing over tube in transvesical position. $D$, video telescope inside abdominal cavity, inserted through over tube.

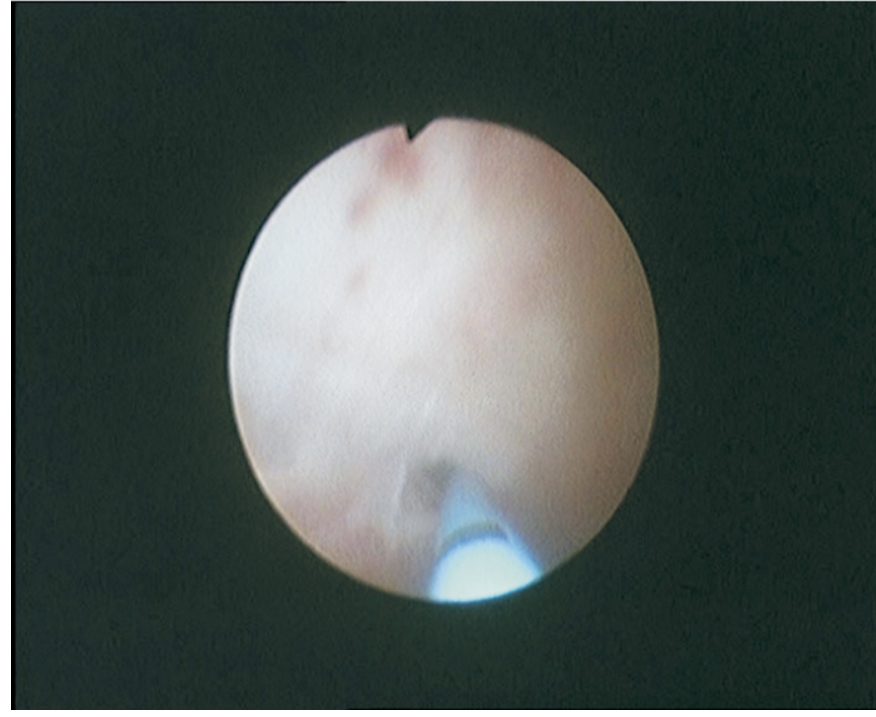

FIG. 2. Endoscopic view of vesical wall perforation with open-ended ureteral catheter

chusetts) enveloped by a flexible over tube. We designed this equipment with a length of $25 \mathrm{~cm}$, internal diameter of 5.5 $\mathrm{mm}$ and wall thickness of $1 \mathrm{~mm}$. Dilator passage over the guidewire and through the bladder wall spared muscle cutting. The ureteroscope was introduced into the peritoneal cavity in the over tube and allowed to create pressure controlled $\mathrm{CO}_{2}$ pneumoperitoneum up to $12 \mathrm{~mm} \mathrm{Hg}$ using an Olympus® UHI-3 Insufflator.

The peritoneal cavity was examined, biopsy specimens were obtained from the liver with an Olympus® A2423 endoscopic biopsy forceps and the falciform ligament was sectioned with an Olympus® A2576 device (fig. 3). The ureteroscope was withdrawn. It was then possible to introduce into the peritoneal cavity a $5 \mathrm{~mm}$ Olympus ${ }^{\circledR}$ EndoEYE $^{\mathrm{TM}}$ video telescope with chip on the tip and a 0-degree direction of view. The endoscope was removed and the peritoneal cavity was decompressed through the over tube. At the end of this procedure the cystoscope was again introduced to observe inner bladder wall morphology. At the end of the operation a $14 \mathrm{Fr}$ Foley catheter was inserted into the bladder and the balloon was inflated with $10 \mathrm{~cm}^{3}$ saline.

At the beginning of this protocol the first 3 animals were sacrificed immediately at the end of the procedure by anesthetic overdose and the peritoneal cavity was examined grossly. In the 5 survival animals oral feeding was started within the following 24 hours. They were evaluated daily and followed for 15 days. The bladder catheter was removed

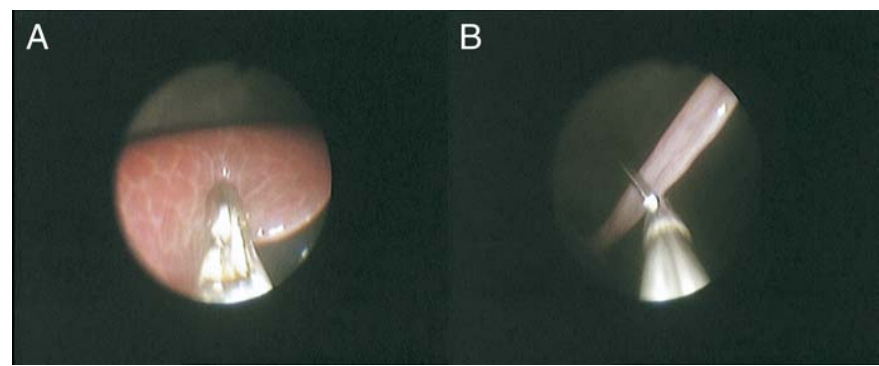

Fig. 3. Representative endoscopic views. $A$, transvesical liver biopsy. $B$, transvesical falciform ligament section. 
4 days after surgery except in 1 animal, in which the vesical catheter exteriorized accidentally 12 hours after surgery. Necropsy was performed at the end of followup with particular attention to the vesical incision site.

\section{RESULTS}

Through a rapid learning curve the first 3 nonsurvival pigs were used to acquire the necessary skills to perform transvesical endoscopic peritoneoscopy. In the subsequent survival animals all procedures involved in creating the vesical hole, including cystoscopy, bladder mucosal incision, vesicotomy and transvesical over tube passage, were performed without complications. The ureteroscope was introduced easily into the peritoneal cavity and $\mathrm{CO}_{2}$ insufflation was performed without incident. The view of the internal organs provided by this instrument was reasonable, mainly for organs of the upper abdomen, including the liver, gallbladder, stomach, spleen and diaphragm. The length of the ureteroscope used allowed us to perform simple surgical procedures, such as liver biopsy and falciform ligament section, in all animals without difficulty. The over tube allowed us to easily introduce the EndoEYE ${ }^{\mathrm{TM}}$, which provided a detailed view of all intra-abdominal organs (fig. 4). After over tube removal the cystoscope showed obvious signs of contraction, making the vesical hole appear like a puncture hole. Operative time from cystoscope introduction to the completion of surgery was between 20 and 40 minutes.

After recovery from anesthesia the pigs tolerated a regular diet within 24 hours, ate heartily and thrived for the next 14 days. Any adverse events occurred in the survival period. Until postoperative day 4 the pigs tolerated the bladder catheter well. After its removal the pigs voided normally.

Necropsy 15 days after surgery revealed complete healing of the bladder wall incision. The vesical perforation location was detected on the inner surface of the bladder as a small dimple (fig. 5). At necropsy the liver biopsy sites were completely healed. There were no signs of infection or adhesions in the peritoneal cavity.

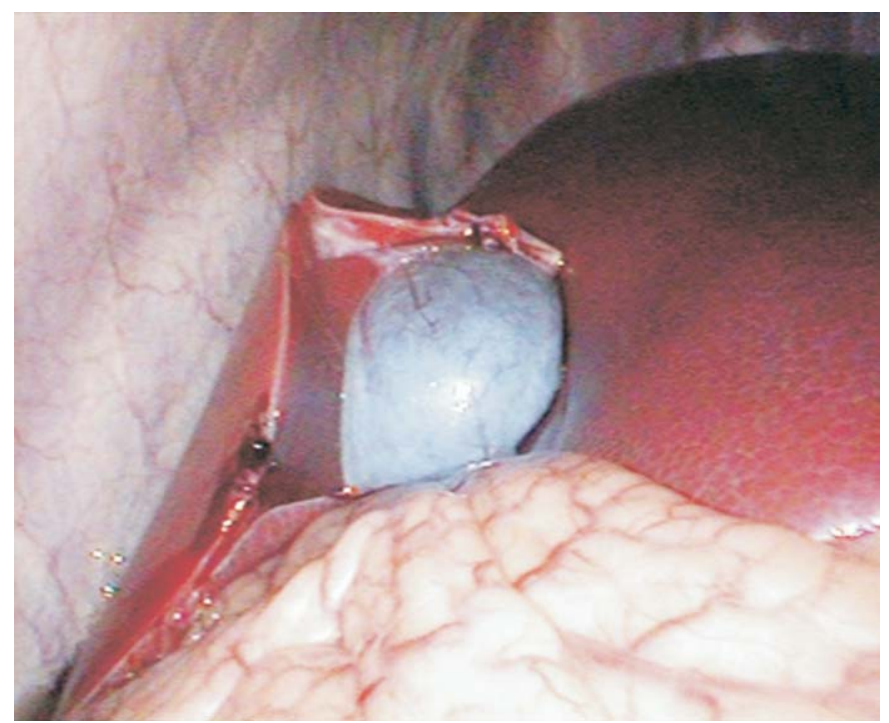

FIG. 4. Endoscopic view of upper abdominal organs, that is liver and gallbladder, provided by video telescope with chip on tip inserted through over tube. Over gallbladder note sectioned falciform ligament. Bleeding point on liver edge represents liver biopsy.

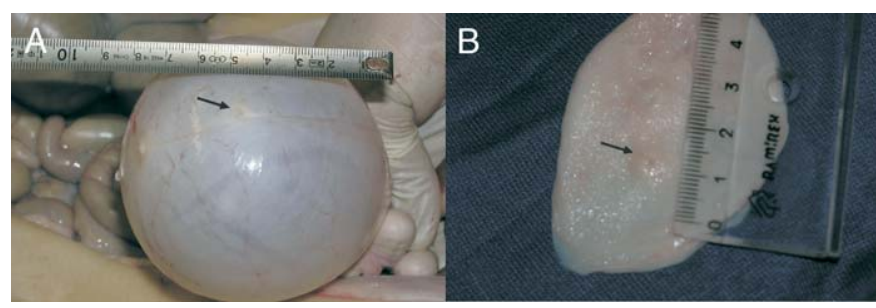

Fig. 5. Bladder necropsy. $A$, external surface of air filled bladder with closed hole (arrow). $B$, inner bladder surface with small residual dimple at surgical perforation site (arrow).

\section{DISCUSSION}

Since Kelling used a Nitze cystoscope combined with pneumoperitoneum to examine the peritoneal cavity in a dog in 1902, many advances in endoscopic intra-abdominal surgery have occurred. ${ }^{1}$ In fact, the rationale for the current study comes from constant technological advances making better and more reliable instruments available for endoscopic procedures and from the recent, unexpected success of transgastric surgery described in porcine models. ${ }^{2-6}$ These facts led us to predict that an additional transvisceral approach positioned diametrically opposed to the stomach might be helpful for performing complex intraperitoneal endoscopic procedures.

In this sequence we tested the possibility of creating a transvesical $5 \mathrm{~mm}$ port. Our study reveals that it was easy to perform it while handling the current instruments used for urological purposes. We began all procedures with a cystoscope, mainly to facilitate the perception of anatomy, which in the porcine model is different from that in humans since the urethra opens in a urogenital sinus. Additionally, the larger vision field supplied by the cystoscope compared to a ureteroscope allowed us to easily define the bladder point to perforate. We selected this point in the ventral wall to achieve a peritoneal cavity above the level of the bowel loops. Perforation of the bladder wall with the open-ended ureteral catheter was rapid, efficient and safe. It should be emphasized that the incision performed in the bladder mucosa was essential to fix the catheter tip and prevent easy looping of the catheter inside the bladder. To date with this technique we have never injured any bowel loop since this catheter is nontraumatic.

Passage of the over tube with the ureteral dilator guided by the guidewire was also an easy operation. The ureteroscope working channel allowed the creation of pressure controlled $\mathrm{CO}_{2}$ pneumoperitoneum. Although it has limited width, the view provided by a $9.8 \mathrm{Fr}$ ureteroscope allowed us to perform simple procedures, such as liver biopsies and falciform ligament section.

In our point of view a transvesical $5 \mathrm{~mm}$ port could be particularly useful for complementing other transvisceral ports. In fact, a group that performed complex abdominal procedures through a transgastric pathway detected problems in working with decreased triangulation and many times with retroflexion. ${ }^{4}$ The position of a transvesical port could easily overcome some of these limitations with additional advantages for performing those complex operations. In fact, it easily achieved by introducing a rigid $5 \mathrm{~mm}$ instrument or a video telescope, which can be particularly useful in those procedures. It should be emphasized that the transvesical port provides frontal access to the upper ab- 
dominal organs, allowing better instrument position for working on these organs.

During creation of the vesical hole in our protocol the risk of bowel perforation was never neglected. However, in our limited number of experiments we never damaged any bowel loop. The explanation of these results might be related to several aspects. 1) Bowel loops in contact with the bladder wall are free into the abdomen, likely making them run ahead of the ureteral catheter as it perforates the vesical wall. 2) Subsequent procedures to position the over tube were performed while guided by the hydrophilic guidewire with atraumatic equipment.

Vesical perforation has potential complications, such as peritoneal urine leakage with secondary infection (peritonitis). This commonly occurs as a delayed complication of undiagnosed vesical perforation in trauma or pathological bladder conditions, such as neoplasms. ${ }^{12}$ In fact, we had reservations about leaving the transvesical entry site unclosed. Interestingly we did not note any of those complications in our study, suggesting that suture or approximation of the vesical wall was unnecessary. In our understanding this might be related to various reasons. 1) The technique that we used to perforate the bladder wall spared muscle fiber section. It seems that muscle fibers contract around the hole immediately after over tube removal. At the end of the procedure we could see through the cystoscope that the vesical hole became immediately and virtually closed. 2) Bladder decompression provided by the vesical catheter surely helped prevent peritoneal leakage, enhancing all healing processes. In this regard we stress that in the last pig in our study the vesical catheter exteriorized within 12 hours after surgery and even in these circumstances no intra-abdominal complications were observed. 3) We did not deal with abnormal bladder tissue invaded by neoplasm or infiltrated by inflammatory cells.

The possibility of installing a $5 \mathrm{~mm}$ port, which might allow the introduction of a $5 \mathrm{~mm}$ rigid instrument or a video telescope with chip on the tip offering an excellent view of the upper abdominal organs, makes this approach appealing for a combination with a transgastric pathway for future intra-abdominal scarless surgery. In addition to cosmesis, we predict that these procedures performed via a transvisceral pathway may have easier recovery than traditional surgery. We think that postoperative pain might be significantly decreased since the density of pain receptors on the viscera is significantly lower than that in the abdominal wall.

\section{CONCLUSIONS}

This study demonstrates that the transvesical endoscopic approach to the peritoneal cavity is feasible without any further complications in a porcine model. This series provides encouragement for additional preclinical studies of transvesical surgery with or without combinations with other natural orifice approaches to design new intra-abdominal scarless procedures in what seems to be the thirdgeneration surgery.

\section{ACKNOWLEDGMENTS}

André Oliveira and Mário Moita, Olympus-Portugal and Manuel Silva contributed to this project.

\section{REFERENCES}

1. Harrell, A. G. and Heniford, T.: Minimally invasive abdominal surgery: lux et veritas past, present, and future. Am J Surg, 190: 239,2005

2. Kalloo, A. N., Singh, V. K., Jagannath, S. B., Niiyama, H., Hill, S. L., Vaughn, C. A. et al: Flexible transgastric peritoneoscopy: a novel approach to diagnostic and therapeutic interventions in the peritoneal cavity. Gastrointest Endosc, 60: 114,2004

3. Jagannath, S. B., Kantsevoy, S. V., Vaughn, C. A., Chung, S. S. C., Cotton, P. B., Gostout, C. J. et al: Per-oral transgastric ligation of fallopian tubes with long-term survival in a porcine model. Gastrointest Endosc, 61: 449, 2005

4. Park, P. O., Bergstrom, M., Ikeda, K., Fritscher-Ravens, A. and Swain, P.: Experimental studies of transgastric gallbladder surgery: cholecystectomy and cholecystogastric anastomosis (videos). Gastrointest Endosc, 61: 601, 2005

5. Kantsevoy, S. V., Jagannath, S. B., Niiyama, H., Chung, S. S. C., Cotton, P. B., Gostout, C. J. et al: Endoscopic gastrojejunostomy with survival in a porcine model. Gastrointest Endosc, 62: 287, 2005

6. Wagh, M. S., Merrifield, B. F. and Thompson, C. C.: Endoscopic transgastric abdominal exploration and organ resection: initial experience in a porcine model. Clin Gastroenterol Hepatol, 3: 892, 2005

7. Ponsky, J. L.: Gastroenterologists as surgeons: what they need to know. Gastrointest Endosc, 61: 454, 2005

8. Hochberger, J. and Lamade, W.: Transgastric surgery in the abdomen: the dawn of a new era? Gastrointest Endosc, 62: 293, 2005

9. Vitale, G. C., Davis, B. R. and Tan, T. C.: The advancing art and science of endoscopy. Am J Surg, 190: 228, 2005

10. Weickert, U., Jakobs, R. and Riemann, J. F.: Diagnostic laparoscopy. Endoscopy, 37: 33, 2005

11. Liu, R., Chand, B. and Ponsky, J.: The future of surgical endoscopy. Endoscopy, 37: 38, 2005

12. Cass, A. S. and Luxenberg, M.: Features of 164 bladder ruptures. J Urol, 138: 743, 1987 